\title{
Electrochemical deposition of polypyrrole nanolayers on discontinuous ultrathin gold films
}

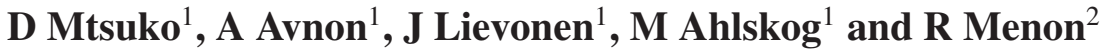 \\ ${ }^{1}$ Nanoscience Center, University of Jyväskylä, POB 35 (YFL), FI-40014, Finland \\ ${ }^{2}$ Department of Physics, Indian Institute of Science, Bangalore, Karnataka 560 012, India
}

Received 16 October 2007, in final form 21 December 2007

Published 20 February 2008

Online at stacks.iop.org/Nano/19/125304

\begin{abstract}
Ultrathin layers of polypyrrole (PPy) were electrochemically grown between microelectrodes on a $\mathrm{Si} / \mathrm{SiO}_{2}$ substrate. Conducting nanolayers of PPy are directly grown onto ultrathin discontinuous gold $(\mathrm{Au})$ film between the microelectrodes, with thicknesses in the range $10-100 \mathrm{~nm}$. The system therefore forms a novel (PPy/Au) nanocomposite conductor. Atomic force microscopy (AFM) imaging and conductivity measurements indicate that at all thicknesses a relatively uniform film is formed but with significant roughness that reflects the roughness of the metallic island layer. In PPy/Au films with thickness $\sim 10 \mathrm{~nm}$, the small barriers around the gold islands dominate the conduction, and as the film thickness increases to $100 \mathrm{~nm}$ the intrinsic conductivity of highly doped PPy dominates the charge transport.
\end{abstract}

(Some figures in this article are in colour only in the electronic version)

\section{Introduction}

Typically, conducting polymers (CPs) are $\pi$-conjugated carbon chains that are semiconducting in their pristine form and can be doped to increase the bulk conductivities in the range from 1 to $10^{4} \mathrm{~S} \mathrm{~cm}^{-1}$, depending on quality, orientation, and other factors [1]. The theoretical upper limit to the conductivity is still a matter of debate. During the three decades of intensive research in this class of materials, much of the interest has been towards topics such as the photophysical properties of the pristine form or the synthesis of a processable and moderately well conducting polymer. These choices reflect to a large degree the current view of what are the most promising widespread future applications of conducting polymers (LEDs, printable electronics, etc).

In the wider research field of nanoscale science, including mesoscopic physics, conducting polymers have played a minor role. Instead, carbon-based materials have above all been represented by the carbon nanotube and lately also by single graphene layers. One practical reason, among many others, for this state of affairs lies in the very different approach to the preparation and processing of $\mathrm{CPs}$ versus carbon nanotubes. It is relatively easy with modern electron and scanning probe microscopes to image and manipulate single carbon nanotubes.
On the other hand, though CPs can be easily prepared, often in soluble forms, it is difficult to fabricate well-defined functional nanostructures from CPs.

Among the relatively few works on the fabrication of mesoscopic or nanoscale CP structures, one may mention CP lines deposited by electrochemical dip-pen nanolithography [2], polypyrrole [3] and polyaniline nanotubes [4], and polyacetylene nanofibers [5]. A further category on its own is the electrochemical growth of a CP onto microelectrodes [6], in which interelectrode separations up to microns can be bridged by the polymer. We chose electrochemical synthesis because it provides better control of the doping level of the CP than chemical synthesis and can yield CPs of very high conductivity. However, electrochemical synthesis is generally limited to conducting substrates and, for example, it is considered unsuitable for the preparation of thin-film field-effect transistors [22] on insulating substrates.

With modern methods it is possible to produce gaps of only a few nanometers between two microelectrodes on an insulating substrate. If these two electrodes are connected together to act as a single electrode in an electrochemical polymer growth process, the gap is quickly bridged and an interesting nanoscale metal-CP-metal junction is formed [7]. Large fluctuations in the conductance of these junctions have 
(a)

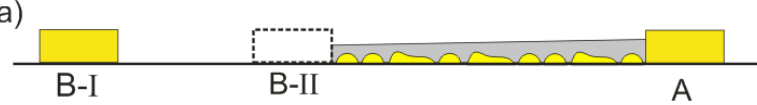

(b)

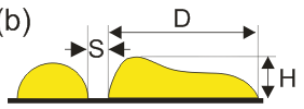

(c)

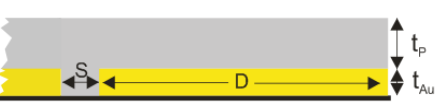

Figure 1. (a) Cross-sectional scheme of the polymerization structure. Electrodes A and B are the anode and cathode, respectively. The gray illustrates the polymer grown on the gold island layer. The placement of electrode B can be according to configuration I or II. (b) Gold islands pictured with definitions of morphological quantities.

(c) Model for conduction in structure (a).

been observed, and were attributed to switching of redox states in individual polymer chains or nanoscale domains in the polymer [8].

By hydrophobic pretreatment of electrodes on an insulating substrate or using localized electric fields, some researchers have been able to promote lateral growth of CPs on insulating substrates [23-25]. The films can be very thin and, moreover, such growth can be made to follow a constrained pattern [26] of submicron dimensions. One drawback with these techniques has been the low conductivity (relative to bulk $\mathrm{CP}$ ) of the resulting polymer structures.

In this work we describe a new way to fabricate electrochemically mesoscopic or nanoscale structures from CPs. The method utilizes patterns of thin, discontinuous, metal films to promote lateral electrochemical growth of the CP. The patterns can be made very small and this also provides an opportunity of studying charge transport in CPs over nanoscale distances corresponding to the inter-island gaps of the metal films.

\subsection{Island layer-based electrochemical growth of polymer}

We have developed a novel configuration for growing thin films of $\mathrm{CP}$ in micron-scale, and even submicron-scale, structures via anodic oxidation, as is often the case. The central idea is to connect the anode to a small patch of discontinuous film of gold, consisting of separated metallic islands. The island layer stretches from the anode towards the cathode. Figure 1(a) shows schematically a cross section of such an island layer and the main parameters describing its structure. Depending on the nominal thickness of the deposited metal, the inter-island separation $(S)$ and the size of the islands varies within a few nanometers.

We have fabricated two different configurations of the systems, I and II, as shown in figure 1(a). It is apparent that in the latter the island layer reaches all the way from the anode, electrode $\mathrm{A}$, towards the cathode, electrode $\mathrm{B}$, while in the former a gap is left between the island layer and electrode B. As the electrodes are covered with a small electrolyte droplet consisting of the monomer and the dopant, the polymer starts to grow from electrode A but extends rapidly from island to island towards B, as the potential is turned on. In configuration II the electropolymerization stops automatically as soon as the deposited polymer bridges the anode and the cathode, and thus diminishes the potential drop at this location. As is shown in this work, the result is a very thin, relatively uniform composite film of a conducting polymer that embeds the gold nanoclusters in between the electrodes. Moreover, the lateral dimensions of the film are accurately determined as those of the island layer. Although somewhat similar work in other systems such as coating a metallic island layer with an oxide [9] or with undoped semiconducting polymer films [10] has been reported, the fabrication procedure in our work is rather different. The $\mathrm{CP}$ used in this work is polypyrrole (PPy), with quite well-known properties and methods for electrochemical preparation [11].

\subsection{Discontinuous metal films}

Metal islands are formed on $\mathrm{Si} / \mathrm{SiO}_{2}$ surfaces by the standard evaporation techniques of deposition. Gold on silicon oxide, which is used in this work, has been studied over the years by several groups $[12,13]$. At very small deposited thicknesses, gold forms small irregularly shaped but compact islands with separations on the order of $10 \mathrm{~nm}$, when enough has been deposited (1-2 nm) for observation by scanning electron microscopy (SEM). As more gold is deposited, the islands become interconnected by percolation to form very irregular clusters. The film becomes continuous only when the clusters merge together, at the percolation threshold. Before this happens the clusters may become micron sized, and sustain a typical distance between them of a few nanometers, which means that a growing island layer is electrically insulating for DC conduction. Close to the percolation threshold, tunneling conduction emerges, but the transition from insulating to fully metallic conduction is very sharp. The electronic transport properties of such discontinuous metal films have been thoroughly investigated by several groups [14, 15]. More recently, the optical field distributions in films close to the percolation threshold has attracted some interest. Measurements with a scanning near-field optical microscope have shown enormous local field enhancements due to the localization of surface plasmons [16].

\section{Experimental details}

Pieces of Si wafers, with thermally grown $\sim 500 \mathrm{~nm}$ thick $\mathrm{SiO}_{2}$ were used as substrates for our structures. Microelectrode structures consisting of four adjacent electrodes of $20 \mathrm{~nm}$ thick gold (with $2 \mathrm{~nm}$ Ti sticking layer) were fabricated with e-beam lithography and lift-off techniques. The electrodes were consecutively labeled as A, C1, C2, and B (defined in figures 1(a) and 3(a)). The chip was electrically connected via bonding. The wider sections of the electrodes leading to the bonding pads were made long enough so that a tiny droplet of the electropolymerization solution could be placed on the microelectrodes without having the liquid in touch with the bonding wires of aluminum. A second step of e-beam lithography was undertaken to connect some of the microelectrodes with a rectangular micron-sized ultrathin $\mathrm{Au}$ film. This discontinuous film, morphologically an 

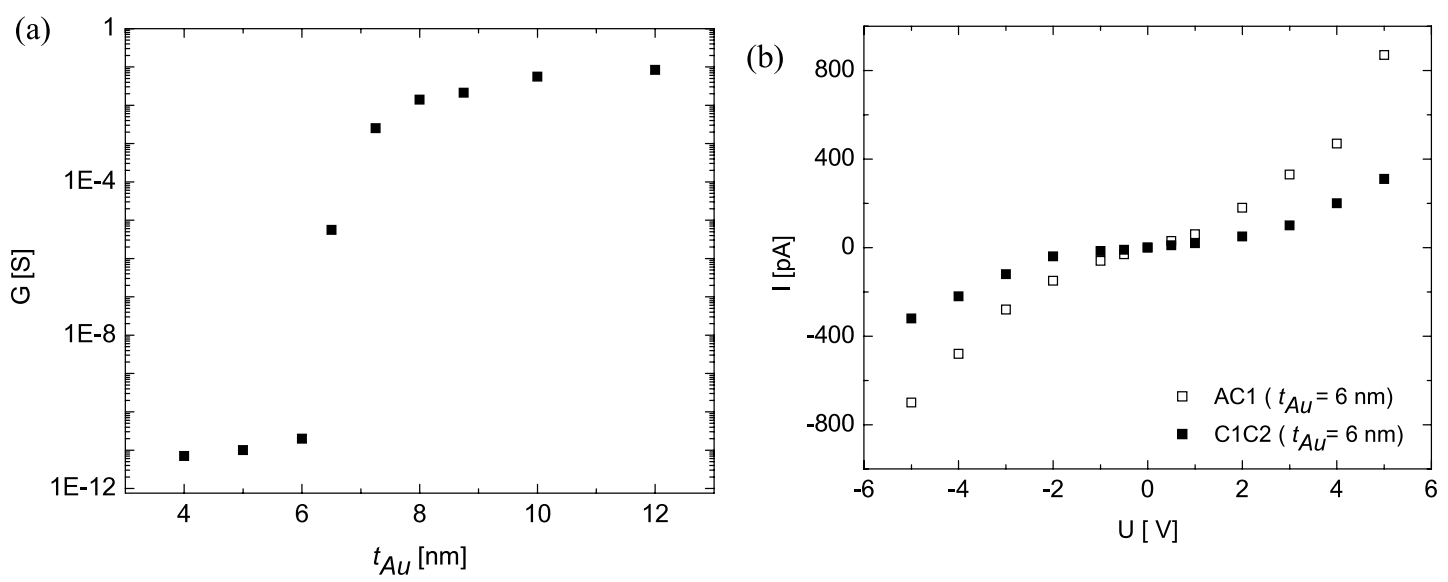

(c)

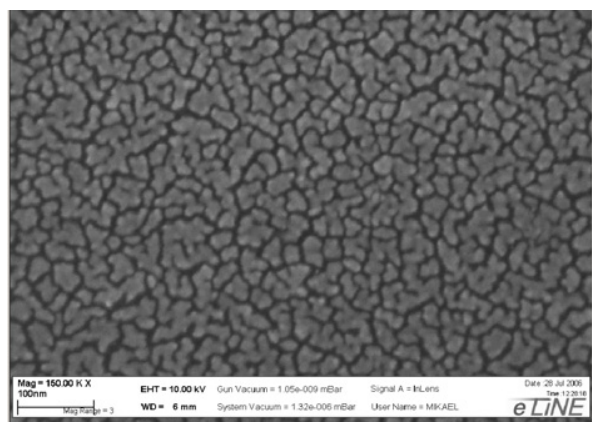

Figure 2. (a) Resistance versus nominal thickness of gold thin films. (b) Test of the leakage current through different sections of an island layer in the type of structures used in this work. The nominal thickness of the film is $6 \mathrm{~nm}$ and the dimensions of the film sections are $1 \times 3 \mu \mathrm{m}$ (width $\times$ length) in both cases. (c) SEM image of a $6 \mathrm{~nm}$ (nominal thickness) gold layer.

'island layer', was deposited with a UHV electron beam evaporator at a rate of deposition of $1 \AA \mathrm{s}^{-1}$, and with careful monitoring of the timing of the shutters that control the metal deposition. No sticking layer was used with the deposition of the island layer. These island structures are quite susceptible to serious damage by electrostatic discharges and were therefore constantly protected after fabrication with different measures, such as shorting the terminals and careful grounding.

The electropolymerization solution consists of $0.1 \mathrm{M}$ pyrrole monomer $\left(\mathrm{C}_{4} \mathrm{H}_{5} \mathrm{~N}\right)$ from Aldrich Co., and $0.05 \mathrm{M}$ tetrabutylammonium hexafluorophosphate $\left(\mathrm{TBA} \mathrm{PF}_{6}\right)$ salt in propylene carbonate solvent. A small droplet was placed on the sample to span the A (anode) and B (cathode) electrodes, and a voltage of 1.5-4 V was applied between these two. Current densities within the range $0.2-30 \mathrm{~mA} \mathrm{~cm}^{-2}$ were obtained. The polymerization started at the anode, spreading through the island layer towards the cathode. For configuration II, in which the island layer is in contact with both the anode and cathode, the polymerization stops automatically as soon as the PPy growth reaches the cathode-when the potential difference between the anode and the cathode diminishesand within this duration the thickness of the polymer in the region $\mathrm{A}-\mathrm{C} 1$ can grow up to $100 \mathrm{~nm}$. To obtain thinner layers of PPy the polymerization had to be stopped earlier, before the polymer layer bridged the anode and the cathode. Typically the polymerization process is stopped within $15 \mathrm{~s}$.

\section{Results and discussion}

\subsection{Island layer characteristics}

The formation of islands ( $\mathrm{Au}$ on $\mathrm{SiO}_{2}$ substrate) and the eventual coalescence to a continuous film on macroscopic scales depends to some degree on the deposition conditions. We examined the resistance versus measured nominal $\mathrm{Au}$ thickness of films evaporated onto $\mathrm{Si} / \mathrm{SiO}_{2}$ substrates, as shown in figure 2(a).

In our setup the sharp conductance transition occurs at a measured $\mathrm{Au}$ thickness of $7 \mathrm{~nm}$. The data were well reproducible, and refer to the zero-bias resistance, measured in the millivolt range. Figure 2(b) shows measurements of leakage currents at higher voltages through island layer structures (before electropolymerization) of the type actually used in this work. The Au island films between the electrodes were always checked to be insulating before polymerization, with a zero-bias resistance $\sim 100 \mathrm{G} \Omega$.

Above thicknesses of $2-3 \mathrm{~nm}$, the islands become quite irregular in shape. An SEM image of a film of thickness $6 \mathrm{~nm}$, which was used in this work, is shown in figure 2(c). A rough analysis of the island morphology from the SEM and AFM images shows that the inter-island separation $S$ changes considerably less than the island (or cluster) size, which grows drastically. Thus the ratio $D / S$ changes very strongly near the transition. The height $(H)$ of the islands as measured with 

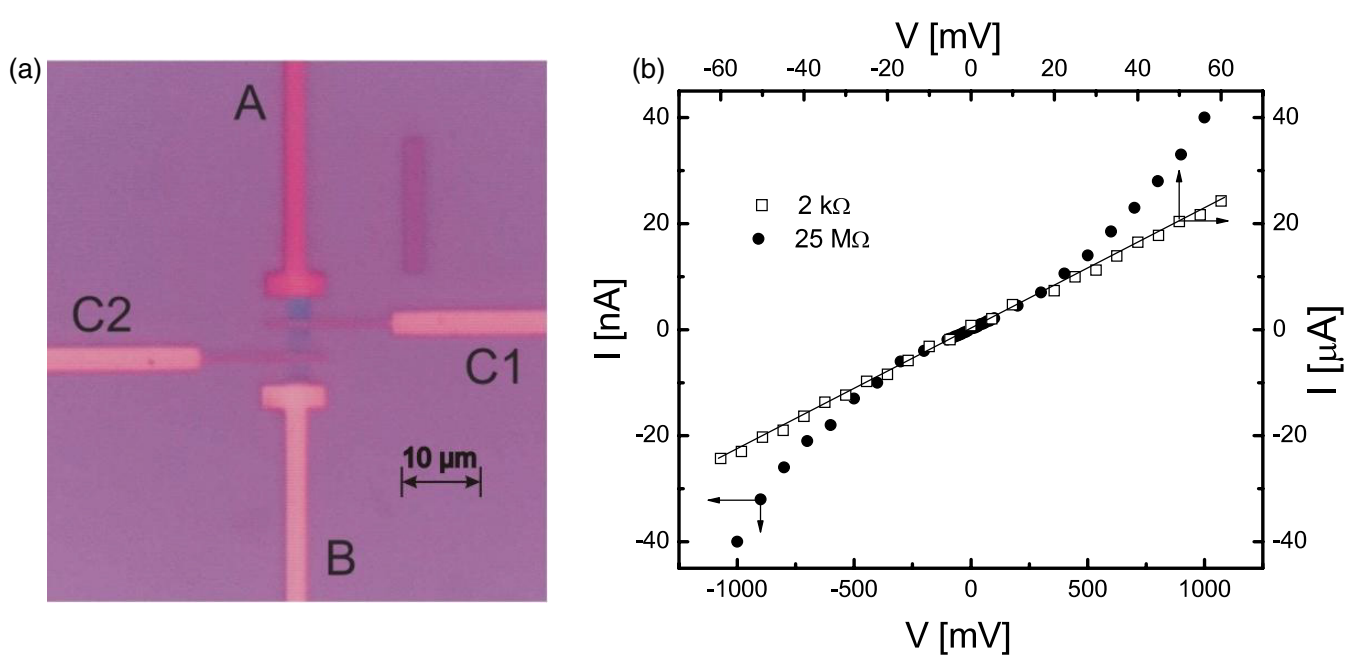

Figure 3. (a) Photograph of a structure after polymerization. The island layer width is $3 \mu \mathrm{m}$, and it is of configuration II (as defined in figure 1). A and B are the anode and cathode, respectively, and C1 and C2 are electrodes used to measure the resistance of the composite film. A separate and identical non-connected island layer to the right of A electrode A displays the color change (online) due to the polymerization. Polymer has also grown on electrode A. (b) Current $(I)$ versus voltage $(U)$ for two polymerized structures of different resistance levels.

Table 1. Dimensions of the island layers onto which PPy is polymerized. The configuration (figure 1(a)), length $(L)$ between different sections (figure 3 ), width $(w)$, and nominal thickness $\left(t_{\mathrm{Au}}\right)$ are presented.

\begin{tabular}{lllll}
\hline Sample & Config. & $L(\mu \mathrm{m}) \mathrm{AC}_{1} / \mathrm{C}_{1} \mathrm{C}_{2} / \mathrm{C}_{2} \mathrm{~B}$ & $w(\mu \mathrm{m})$ & $t_{\mathrm{Au}}(\mathrm{nm})$ \\
\hline $\mathrm{A}$ & $\mathrm{I}$ & $3 / 5 / 7$ & 5 & 6 \\
$\mathrm{~B}$ & $\mathrm{I}$ & $5 / 5 / 5$ & 5 & 6 \\
$\mathrm{C}$ & $\mathrm{II}$ & $3 / 3 / 2$ & 1.5 & 5 \\
$\mathrm{D}$ & $\mathrm{II}$ & $3 / 3 / 2$ & 1.5 & 6 \\
$\mathrm{E}$ & $\mathrm{II}$ & $3 / 3 / 2$ & 1.5 & 6 \\
$\mathrm{~F}$ & $\mathrm{II}$ & $3 / 3 / 2$ & 1.5 & 6 \\
\hline
\end{tabular}

AFM was always slightly larger than the nominal thickness measured with the thickness monitor of the UHV evaporator. Our results correspond well with previously reported data on thin gold films [12].

\subsection{Electropolymerization}

Table 1 presents the dimensions of the samples that were polymerized in this work, and figure 3(a) shows the visual results of the polymerization to a $3 \mu \mathrm{m} \times 8 \mu \mathrm{m}$ sized island layer structure. Nearly all the samples in this work were of nominal thickness $6 \mathrm{~nm}$, which had relatively large islands $(\sim 100 \mathrm{~nm})$. The image (figure 3(a)) and AFM images presented later show very clearly the ability of the island layer to guide the polymer growth along its surface, from island to island. Additional results, not shown here, showed that island layers of nominal thickness down to $2 \mathrm{~nm}$ could direct the growth of the polymer, whereas a $1 \mathrm{~nm}$ island layer failed to do it. A $2 \mathrm{~nm}$ film has quite small islands with a $D / S$ ratio $\approx 1$.

Table 2 presents some representative samples with different sizes of the island layers and interelectrode separations. AFM imaging was undertaken to measure the thickness of the PPy/Au layer of the samples as shown in table 2. The thickness was always slightly larger at the A electrode but decreased evenly towards the B electrode.
Figure 3(b) shows measured current-voltage characteristics for two very different samples, with respect to resistance levels. The thicknesses of the $2 \mathrm{k} \Omega$ and $25 \mathrm{M} \Omega$ PPy/Au samples in figure 3 (b) are $\sim 100 \mathrm{~nm}$ and $\sim 20 \mathrm{~nm}$, respectively. Before polymerization the resistance of the gold films was $\sim 100 \mathrm{G} \Omega$.

We imaged a few of our typical island layers with an ultrasharp AFM tip ${ }^{3}$ before and after polymerization, as shown in figure 4. The image of the fresh island layer exhibits very clearly the morphology of gold islands. A thinner polymerized sample, with a total thickness of $45 \mathrm{~nm}$, seemingly still has some of the island morphology beneath. The roughness at this stage is the same in magnitude, $11 \mathrm{~nm}$, as that of the bare island layer. Thicker samples $(120 \mathrm{~nm})$ had clearly increased roughness, a few tens of nanometers.

PPy is among the more stable polymers in the highly doped state; hence its conductivity is quite stable in air for very long duration. Our electropolymerized thin films exhibited quite stable values of resistance. Samples with resistances in the $1-100 \mathrm{k} \Omega$ range stayed constant (less than $10 \%$ variation) at these values for several weeks.

\subsection{Conductivity}

The central question concerning these systems is whether conduction is dominated by the gold island layer, in particular

${ }^{3}$ NanoSensors, SSS-SEIHR. $k=15 \mathrm{~N} \mathrm{~m}^{-1}$. 


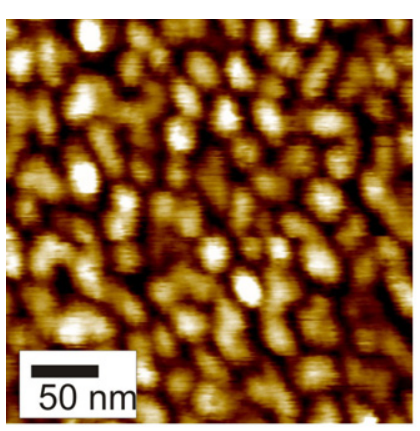

(a)

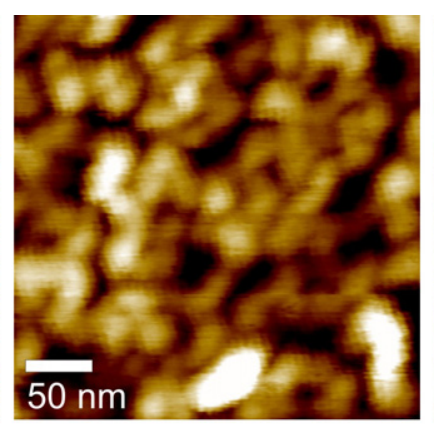

(b)

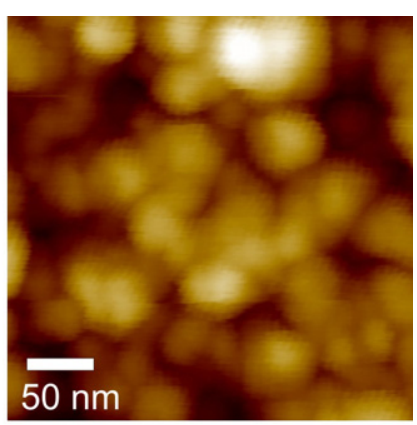

(c)

Figure 4. AFM images taken with an ultrasharp AFM tip. (a) A gold island layer (nominal thickness $6 \mathrm{~nm}$, width $=3 \mu \mathrm{m}$ and zero-bias resistance, $R \sim 100 \mathrm{G} \Omega$ ). (b) Surface of a sample of PPy/Au with total film thickness $\left(t_{\mathrm{Au}}+t_{\mathrm{P}}\right) 45 \mathrm{~nm}$ and $R=770 \mathrm{k} \Omega$. (c) Surface of $\mathrm{PPy} / \mathrm{Au}$ (sample B) with thickness $\left(t_{\mathrm{Au}}+t_{\mathrm{P}}\right) 120 \mathrm{~nm}$ and $R=1.2 \mathrm{k} \Omega$.

Table 2. Samples of table 1 after electropolymerization. Thickness of PPy/Au (i.e. $t_{\mathrm{Au}}+t_{\mathrm{P}}$ ) and (zero-bias) resistance measured at room temperature for the different sections of the island layer.

\begin{tabular}{llll}
\hline Sample & Config. & $t_{\mathrm{Au}}+t_{\mathrm{P}}(\mathrm{nm}) \mathrm{AC}_{1} / \mathrm{C}_{1} \mathrm{C}_{2}$ & $R(\Omega) \mathrm{AC}_{1} / \mathrm{C}_{1} \mathrm{C}_{2}$ \\
\hline $\mathrm{A}$ & $\mathrm{I}$ & $25-75 / 25-40$ & $26 \mathrm{k} / 2.6 \mathrm{M}$ \\
$\mathrm{B}$ & I & $100-120 / 70-90$ & $1.2 \mathrm{k} / 2.6 \mathrm{k}$ \\
$\mathrm{C}$ & II & $14-20 / 10-15$ & $105 \mathrm{k} / 370 \mathrm{k}$ \\
$\mathrm{D}$ & II & $10-25 / 9-12$ & $740 \mathrm{k} / 6.4 \mathrm{M}$ \\
$\mathrm{E}$ & II & Not meas. & $18 \mathrm{M} /-$ \\
$\mathrm{F}$ & II & $50-75 / 35-45$ & $48 \mathrm{k} / 113 \mathrm{k}$ \\
\hline
\end{tabular}

through the small polymer-filled gaps between the islands, or by the doped PPy film itself. In the following we consider the values of room-temperature conductivity and a geometrically simplified model for conduction, as shown in figure 1(c), that averages out the roughness of the polymer films.

To begin with, consider the ideal case when there would be polymer only between the gold islands $\left(t_{\mathrm{P}}=0\right.$ in figure $\left.1(\mathrm{c})\right)$. The conductivity of bulk gold is $5 \times 10^{5} \mathrm{~S} \mathrm{~cm}^{-1}$, while that of highly doped PPy varies in the range $1-10^{3} \mathrm{~S} \mathrm{~cm}^{-1}$ [17], depending on the quality of the polymer. Based on SEM and AFM images, the ratio of $D / S$ is in the range $10-100$ for the 5-6 nm thick (nominal thickness) island layers in this work. We can then assume that the combined resistance of the island layer and the polymer interconnect is dominated by the polymer, even disregarding the interfacial resistance between the polymer and the gold, which is very possibly substantial.

In practice there is a polymer layer above the island layer (i.e. $t_{\mathrm{P}}>0$ ). Restricting oneself to consider ohmic conduction in the model of figure 1(c), the ratio of resistance of the layer of polymer above the island layer $\left(R_{\mathrm{P}}\right)$ or to the resistance of the polymer embedded in the island layer $\left(R_{i}\right)$ reduces to

$$
\frac{R_{\mathrm{P}}}{R_{i}} \approx \frac{D}{S} \frac{t_{\mathrm{Au}}}{t_{\mathrm{P}}}, \quad D \gg S .
$$

The factor $D / S$ strongly depends on the nominal thickness $\left(t_{\mathrm{Au}}\right)$ of the island layer. Within the nominal thickness $\left(t_{\mathrm{Au}}\right.$ : 2-6 nm, where spreading of polymer along the island layer is possible) the weighting factors $D / S$ and $t_{\mathrm{Au}} / t_{\mathrm{P}}$ may easily overtake each other. The resultant resistance ratio, $R_{\mathrm{P}} / R_{i}$, may either be $>1$ or $<1$, and that can determine whether the dominant path of charge transport is through the bulky layer above the island layer or via the underlying polymer-filled island layer. In this work, we concentrated on samples where the nominal gold thickness $t_{\mathrm{Au}}$ equals 5 or $6 \mathrm{~nm}$. In this case we estimate $D / S$ to be in the range $10-100$. The factor $t_{\mathrm{Au}} / t_{\mathrm{P}}$ lies in the range $0.1-1$ for the samples in table 2 , where $t_{\mathrm{P}}$ varies between 100 and $10 \mathrm{~nm}$. In this case the ratio $R_{\mathrm{P}} / R_{i}>1$. One can therefore expect that conduction of the composite system will be dominated by the intrinsic conductivity of the polymer between the islands. The effective resistance of the composite should, therefore, remain $\sim R_{i}$ as the thickness $t_{\mathrm{P}}$ approaches $t_{\mathrm{Au}}$. However, the data in table 2 show very clearly that the total resistance increases much faster than predicted by the ohmic treatment as the thickness $t_{\mathrm{P}}$ of the polymer decreases. In our opinion there are two possible explanations: either the intrinsic quality of the polymer improves with increasing thickness or there are significant electrically resistive barriers between the gold islands and the polymer. We favor the latter case since, considering the very rough morphology of the films, it is likely that conduction in the thinner samples is indeed impeded by small nanoscale barriers.

Therefore, the conduction in thicker samples is dominated by the intrinsic conductivity of the polymer above the island layer. We may then calculate the room-temperature conductivity as $\sigma_{0}=\ell / R_{p} w t_{\mathrm{P}}$. For sample $\mathrm{F}$, one obtains a conductivity of $5-7 \mathrm{~S} \mathrm{~cm}^{-1}$ for a plain film with these dimensions. The best conducting sample, $\mathrm{B}$, yields a conductivity of $75 \mathrm{~S} \mathrm{~cm}^{-1}$. These values are entirely in agreement with previously reported values for PPy electrochemically synthesized at room temperature [18, 19]. In figure 3(b), the more resistive sample exhibits a nonlinear behavior, starting from a voltage of $0.5 \mathrm{~V}$. This voltage corresponds roughly to an inter-island electric field of about $3 \times 10^{4} \mathrm{~V} \mathrm{~cm}^{-1}$, which is enough to produce non-linear phenomena in most conduction mechanisms.

\section{Conclusion}

We conclude that for thicknesses in the $10 \mathrm{~nm}$ range, the conduction through the percolating polymer film is dominated by local barriers in the composite island layer/polymer film, 
while in the $100 \mathrm{~nm}$ range the intrinsic resistivity of the polymer film dominates. The conductive properties of these nanoscale composites of PPy/Au island layer systems promise to exhibit interesting features at low temperatures, as the PPy is just on the insulating side of the metal-insulator transition. The metal-insulator transition has been extensively studied for macroscopic samples of PPy [18, 20, 21]. The present system offers an opportunity to study charge transport in highly doped PPy at the 1-10 nm length scale, corresponding to the interisland separation. Low-temperature transport properties will be addressed in a future publication.

\section{References}

[1] Kiebooms R, Menon R and Lee K 2001 Conducting polymers Handbook of Advanced Electronic and Photonic Materials and Devices vol 8, ed H S Nalwa (New York: Academic)

[2] Maynor B, Filacamo S, Grinstaff M and Liu J $2002 \mathrm{~J}$. Am. Chem. Soc. 124 522-3

[3] Cuenot S, Demoustier-Champagne S and Nysten B 2000 Phys. Rev. Lett. 851690

[4] Long Y et al 2003 Appl. Phys. Lett. 831863

[5] Aleshin A, Lee H, Park Y and Akagi K 2004 Phys. Rev. Lett. 93196601

[6] Zhu S and Swager T 1997 J. Am. Chem. Soc. 11912568

[7] He H, Zhu J, Tao N, Nagahara L, Amlani I and Tsui R 2001 J. Am. Chem. Soc. 1237730

[8] He H, Li X, Tao N, Nagahara L, Amlani I and Tsui R 2003 Phys. Rev. B 68045302
[9] Vullers R, Ahlskog M, Cannaerts M and Van Haesendonck C 2000 Appl. Phys. Lett. 761947

[10] Paloheimo J, Grönberg L and Stubb H 1993 Synth. Met. 55-57 4198-203

[11] Cho S, Song K and Lee J 1998 Recent advances in polypyrrole Handbook of Conducting Polymers 3rd edn, ed T Skotheim and R Reynolds (Florida: CRC Press)

[12] Laibowitz R, Alessandrini E, Guarnieri C and Voss R 1983 J. Vac. Sci. Technol. A 1438

[13] Campbell C 1997 Surf. Sci. Rep. 271

[14] Morris J and Coutts T 1977 Thin Solid Films 473

[15] Yagil Y, Deutscher G and Bergmann D 1992 Phys. Rev. Lett. 691423

[16] Seal K, Nelson M, Ying Z, Genov D, Sarychev A and Shalaev V 2003 Phys. Rev. B 67035318

[17] Lee K and Heeger A 2002 Synth. Met. 128279

[18] Yoon C, Menon R, Moses D and Heeger A 1994 Phys. Rev. B 4910851

[19] Menon R, Yoon C, Moses D and Heeger A 1994 Synth. Met. 6453

[20] Menon R, Vakiparta K, Yoon C, Cao Y, Moses D and Heeger A 1994 Synth. Met. 65167

[21] Ahlskog M, Menon R and Heeger A 1997 J. Phys.: Condens. Matter 94145

[22] Buffon C and Heinzel T 2006 Appl. Phys. Lett. 89012104

[23] Nishizawa M, Shibuya M, Sawaguchi T, Matsue T and Uchida I 1991 J. Phys. Chem. 959042

[24] Nishizawa M, Nozaki H, Kaji H, Kitazume T, Kobayashi N, Ishibashi T and Abe T 2007 Biomaterials 281480

[25] Su M, Fu L, Wu N, Aslam M and Dravid V P 2004 Appl. Phys. Lett. 84828

[26] Woodson M and Liu J 2005 J. Am. Chem. Soc. 1283760 